\title{
Mitochondrial M2 Antibody Measurement
}

National Cancer Institute

\section{Source}

National Cancer Institute. Mitochondrial M2 Antibody Measurement. NCI Thesaurus.

Code 147395.

The determination of the amount of mitochondrial M2 antibody present in a sample. 